Research Article

\title{
Stability of the Moons Orbits in Solar System in the Restricted Three-Body Problem
}

\author{
Sergey V. Ershkov \\ Institute for Time Nature Explorations, M.V. Lomonosov's Moscow State University, Leninskie Gory 1-12, Moscow 119991, Russia \\ Correspondence should be addressed to Sergey V. Ershkov; sergej-ershkov@yandex.ru
}

Received 1 April 2015; Revised 3 June 2015; Accepted 4 June 2015

Academic Editor: Elmetwally Elabbasy

Copyright ( 2015 Sergey V. Ershkov. This is an open access article distributed under the Creative Commons Attribution License, which permits unrestricted use, distribution, and reproduction in any medium, provided the original work is properly cited.

\begin{abstract}
We consider the equations of motion of three-body problem in a Lagrange form (which means a consideration of relative motions of 3 bodies in regard to each other). Analyzing such a system of equations, we consider in detail the case of moon's motion of negligible mass $m_{3}$ around the 2 nd of two giant-bodies $m_{1}, m_{2}$ (which are rotating around their common centre of masses on Kepler's trajectories), the mass of which is assumed to be less than the mass of central body. Under assumptions of R3BP, we obtain the equations of motion which describe the relative mutual motion of the centre of mass of 2 nd giant-body $m_{2}$ (planet) and the centre of mass of 3 rd body (moon) with additional effective mass $\xi \cdot m_{2}$ placed in that centre of mass $\left(\xi \cdot m_{2}+m_{3}\right)$, where $\xi$ is the dimensionless dynamical parameter. They should be rotating around their common centre of masses on Kepler's elliptic orbits. For negligible effective mass $\left(\xi \cdot m_{2}+m_{3}\right)$ it gives the equations of motion which should describe a quasi-elliptic orbit of 3rd body (moon) around the 2nd body $m_{2}$ (planet) for most of the moons of the planets in Solar System.
\end{abstract}

\section{Introduction}

The stability of the motion of the moon is the ancient problem which leading scientists have been trying to solve during last 400 years. A new derivation to estimate such a problem from a point of view of relative motions in restricted three-body problem $(\mathrm{R} 3 \mathrm{BP})$ is proposed here.

Systematic approach to the problem above was suggested earlier in KAM- (Kolmogorov-Arnold-Moser-) theory [1] in which the central KAM-theorem is known to be applied for researches of stability of Solar System in terms of restricted three-body problem [2-5], especially if we consider photogravitational restricted three-body problem [6-8] with additional influence of Yarkovsky effect of nongravitational nature [9].

KAM is the theory of stability of dynamical systems [1] which should solve a very specific question in regard to the stability of orbits of so-called "small bodies" in Solar System, in terms of restricted three-body problem [3]: indeed, dynamics of all the planets is assumed to satisfy restrictions of restricted three-body problem (such as infinitesimal masses and negligible deviations of the main orbital elements).

Nevertheless, KAM also is known to assume the appropriate Hamilton formalism in proof of the central KAMtheorem [1]; the dynamical system is assumed to be Hamilton's system and all the mathematical operations over such a dynamical system are assumed to be associated with a proper Hamilton system.

According to the Bruns theorem [5], there are no other invariants except well-known 10 integrals for three-body problem (including integral of energy and momentum); this is a classical example of Hamilton's system. But in case of restricted three-body problem, there are no other invariants except only one, Jacobian-type integral of motion [3].

Such a contradiction is the main paradox of KAM-theory; it adopts all the restrictions of restricted three-body problem, but nevertheless it proves to use the Hamilton formalism, which assumes the conservation of all other invariants (the integral of energy, momentum, etc.).

To avoid ambiguity, let us consider a relative motion in three-body problem [2]. 


\section{Equations of Motion}

Let us consider the system of ODE for restricted three-body problem in barycentric Cartesian coordinate system, at given initial conditions $[2,3]$ :

$$
\begin{aligned}
& m_{1} \mathbf{q}_{1}^{\prime \prime}=-\gamma\left\{\frac{m_{1} m_{2}\left(\mathbf{q}_{1}-\mathbf{q}_{2}\right)}{\left|\mathbf{q}_{1}-\mathbf{q}_{2}\right|^{3}}+\frac{m_{1} m_{3}\left(\mathbf{q}_{1}-\mathbf{q}_{3}\right)}{\left|\mathbf{q}_{1}-\mathbf{q}_{3}\right|^{3}}\right\}, \\
& m_{2} \mathbf{q}_{2}^{\prime \prime}=-\gamma\left\{\frac{m_{2} m_{1}\left(\mathbf{q}_{2}-\mathbf{q}_{1}\right)}{\left|\mathbf{q}_{2}-\mathbf{q}_{1}\right|^{3}}+\frac{m_{2} m_{3}\left(\mathbf{q}_{2}-\mathbf{q}_{3}\right)}{\left|\mathbf{q}_{2}-\mathbf{q}_{3}\right|^{3}}\right\}, \\
& m_{3} \mathbf{q}_{3}^{\prime \prime}=-\gamma\left\{\frac{m_{3} m_{1}\left(\mathbf{q}_{3}-\mathbf{q}_{1}\right)}{\left|\mathbf{q}_{3}-\mathbf{q}_{1}\right|^{3}}+\frac{m_{3} m_{2}\left(\mathbf{q}_{3}-\mathbf{q}_{2}\right)}{\left|\mathbf{q}_{3}-\mathbf{q}_{2}\right|^{3}}\right\},
\end{aligned}
$$

where $\mathbf{q}_{1}, \mathbf{q}_{2}$, and $\mathbf{q}_{3}$ mean the radius vectors of bodies $m_{1}$, $m_{2}$, and $m_{3}$, respectively; $\gamma$ is the gravitational constant.

The system above could be represented for relative motion of three bodies as shown below (by the proper linear transformations):

$$
\begin{aligned}
& \left(\mathbf{q}_{1}-\mathbf{q}_{2}\right)^{\prime \prime}+\gamma\left(m_{1}+m_{2}\right) \frac{\left(\mathbf{q}_{1}-\mathbf{q}_{2}\right)}{\left|\mathbf{q}_{1}-\mathbf{q}_{2}\right|^{3}} \\
& =\gamma m_{3}\left\{\frac{\left(\mathbf{q}_{3}-\mathbf{q}_{1}\right)}{\left|\mathbf{q}_{3}-\mathbf{q}_{1}\right|^{3}}+\frac{\left(\mathbf{q}_{2}-\mathbf{q}_{3}\right)}{\left|\mathbf{q}_{2}-\mathbf{q}_{3}\right|^{3}}\right\}, \\
& \left(\mathbf{q}_{2}-\mathbf{q}_{3}\right)^{\prime \prime}+\gamma\left(m_{2}+m_{3}\right) \frac{\left(\mathbf{q}_{2}-\mathbf{q}_{3}\right)}{\left|\mathbf{q}_{2}-\mathbf{q}_{3}\right|^{3}} \\
& =\gamma m_{1}\left\{\frac{\left(\mathbf{q}_{3}-\mathbf{q}_{1}\right)}{\left|\mathbf{q}_{3}-\mathbf{q}_{1}\right|^{3}}+\frac{\left(\mathbf{q}_{1}-\mathbf{q}_{2}\right)}{\left|\mathbf{q}_{1}-\mathbf{q}_{2}\right|^{3}}\right\}, \\
& \left(\mathbf{q}_{3}-\mathbf{q}_{1}\right)^{\prime \prime}+\gamma\left(m_{1}+m_{3}\right) \frac{\left(\mathbf{q}_{3}-\mathbf{q}_{1}\right)}{\left|\mathbf{q}_{3}-\mathbf{q}_{1}\right|^{3}} \\
& =\gamma m_{2}\left\{\frac{\left(\mathbf{q}_{1}-\mathbf{q}_{2}\right)}{\left|\mathbf{q}_{1}-\mathbf{q}_{2}\right|^{3}}+\frac{\left(\mathbf{q}_{2}-\mathbf{q}_{3}\right)}{\left|\mathbf{q}_{2}-\mathbf{q}_{3}\right|^{3}}\right\} .
\end{aligned}
$$

Let us designate the following:

$$
\begin{aligned}
& \mathbf{R}_{1,2}=\left(\mathbf{q}_{1}-\mathbf{q}_{2}\right), \\
& \mathbf{R}_{2,3}=\left(\mathbf{q}_{2}-\mathbf{q}_{3}\right), \\
& \mathbf{R}_{3,1}=\left(\mathbf{q}_{3}-\mathbf{q}_{1}\right) .
\end{aligned}
$$

Using of $(*)$ above, let us transform the previous system to another form:

$$
\begin{aligned}
& \mathbf{R}_{1,2}^{\prime \prime}+\gamma\left(m_{1}+m_{2}\right) \frac{\mathbf{R}_{1,2}}{\left|\mathbf{R}_{1,2}\right|^{3}}=\gamma m_{3}\left\{\frac{\mathbf{R}_{3,1}}{\left|\mathbf{R}_{3,1}\right|^{3}}+\frac{\mathbf{R}_{2,3}}{\left|\mathbf{R}_{2,3}\right|^{3}}\right\}, \\
& \mathbf{R}_{2,3}^{\prime \prime}+\gamma\left(m_{2}+m_{3}\right) \frac{\mathbf{R}_{2,3}}{\left|\mathbf{R}_{2,3}\right|^{3}}=\gamma m_{1}\left\{\frac{\mathbf{R}_{1,2}}{\left|\mathbf{R}_{1,2}\right|^{3}}+\frac{\mathbf{R}_{3,1}}{\left|\mathbf{R}_{3,1}\right|^{3}}\right\}, \\
& \mathbf{R}_{3,1}^{\prime \prime}+\gamma\left(m_{1}+m_{3}\right) \frac{\mathbf{R}_{3,1}}{\left|\mathbf{R}_{3,1}\right|^{3}}=\gamma m_{2}\left\{\frac{\mathbf{R}_{2,3}}{\left|\mathbf{R}_{2,3}\right|^{3}}+\frac{\mathbf{R}_{1,2}}{\left|\mathbf{R}_{1,2}\right|^{3}}\right\} .
\end{aligned}
$$

Analysing system (3) we should note that if we sum all the above equations one to each other it would lead us to the result below:

$$
\mathbf{R}_{1,2}^{\prime \prime}+\mathbf{R}_{2,3}^{\prime \prime}+\mathbf{R}_{3,1}^{\prime \prime}=0
$$

If we also sum all the equalities $(*)$ one to each other, we should obtain

$$
\mathbf{R}_{1,2}+\mathbf{R}_{2,3}+\mathbf{R}_{3,1}=0 \text {. }
$$

Under assumption of restricted three-body problem, we assume that the mass of small 3rd body $m_{3} \ll m_{1}, m_{2}$, respectively; besides, for the case of moving of small 3rd body $m_{3}$ as a moon around the 2 nd body $m_{2}$, let us additionally assume $\left|\mathbf{R}_{2,3}\right| \ll\left|\mathbf{R}_{1,2}\right|$.

So taking into consideration $(* *)$, we obtain from system (3) the following:

$$
\begin{aligned}
& \mathbf{R}_{1,2}^{\prime \prime}+\gamma\left(m_{1}+m_{2}\right) \frac{\mathbf{R}_{1,2}}{\left|\mathbf{R}_{1,2}\right|^{3}}=0, \\
& \mathbf{R}_{2,3}^{\prime \prime}+\gamma\left(m_{2}+m_{3}\right) \frac{\mathbf{R}_{2,3}}{\left|\mathbf{R}_{2,3}\right|^{3}} \\
& =\gamma m_{1}\left\{\frac{\mathbf{R}_{1,2}}{\left|\mathbf{R}_{1,2}\right|^{3}}-\frac{\left(\mathbf{R}_{1,2}+\mathbf{R}_{2,3}\right)}{\left|\mathbf{R}_{1,2}+\mathbf{R}_{2,3}\right|^{3}}\right\}, \\
& \mathbf{R}_{1,2}+\mathbf{R}_{2,3}+\mathbf{R}_{3,1}=0,
\end{aligned}
$$

where the 1st equation of (5) describes the relative motion of 2 massive bodies (which are rotating around their common centre of masses on Kepler's trajectories); the 2nd describes the orbit of small 3rd body $m_{3}$ (moon) relative to the 2 nd body $m_{2}$ (planet), for which we could obtain according to the trigonometric "Law of Cosines" [10]:

$$
\begin{aligned}
\mathbf{R}_{2,3}^{\prime \prime} & +\gamma\left(m_{2}+m_{3}\right) \frac{\mathbf{R}_{2,3}}{\left|\mathbf{R}_{2,3}\right|^{3}} \\
& +\frac{\gamma m_{1}}{\left|\mathbf{R}_{1,2}\right|^{3}}\left(1+3 \cos \alpha \frac{\left|\mathbf{R}_{2,3}\right|}{\left|\mathbf{R}_{1,2}\right|}\right) \mathbf{R}_{2,3} \\
\cong & -3 \cos \alpha\left(\frac{\gamma m_{1}}{\left|\mathbf{R}_{1,2}\right|^{3}} \mathbf{R}_{1,2}\right) \frac{\left|\mathbf{R}_{2,3}\right|}{\left|\mathbf{R}_{1,2}\right|}
\end{aligned}
$$

where $\alpha$ is the angle between the radius-vectors $\mathbf{R}_{2,3}$ and $\mathbf{R}_{1,2}$.

Equation (6) could be simplified under the additional assumption above $\left|\mathbf{R}_{2,3}\right| \ll\left|\mathbf{R}_{1,2}\right|$ for restricted mutual motions of bodies $m_{1}$ and $m_{2}$ in R3BP [3] as below:

$$
\mathbf{R}_{2,3}^{\prime \prime}+\left(\frac{\gamma\left(m_{2}+m_{3}\right)}{\left|\mathbf{R}_{2,3}\right|^{3}}+\frac{\gamma m_{1}}{\left|\mathbf{R}_{1,2}\right|^{3}}\right) \cdot \mathbf{R}_{2,3}=0 .
$$


Moreover, if we present (7) in the form below

$$
\begin{aligned}
\mathbf{R}_{2,3}^{\prime \prime}+\gamma(1+\xi+\eta) \cdot m_{2} & \cdot \frac{\mathbf{R}_{2,3}}{\left|\mathbf{R}_{2,3}\right|^{3}}=0, \\
\xi & =\left(\frac{m_{1}}{m_{2}} \cdot \frac{\left|\mathbf{R}_{2,3}\right|^{3}}{\left|\mathbf{R}_{1,2}\right|^{3}}\right), \eta=\left(\frac{m_{3}}{m_{2}}\right),
\end{aligned}
$$

then (8) describes the relative motion of the centre of mass of 2 nd giant-body $m_{2}$ (planet) and the centre of mass of 3rd body (moon) with the effective mass $\left(\xi \cdot m_{2}+m_{3}\right)$, which are rotating around their common centre of masses on the stable Kepler's elliptic trajectories.

Besides, if the dimensionless parameters $\xi, \eta \rightarrow 0$, then (8) should describe a quasi-circle motion of 3rd body (moon) around the 2 nd body $m_{2}$ (planet).

\section{The Comparison of the Moons in Solar System}

As we can see from (8), $\xi$ is the key parameter which determines the character of moving of the small 3rd body $m_{3}$ (the moon) relative to the 2 nd body $m_{2}$ (planet). Let us compare such a parameter for all considerable known cases of orbital moving of the moons in Solar System [11] (Table 1).

\section{Discussion}

As we can see from Table 1, the dimensionless key parameter $\xi$, which determines the character of moving of the small 3rd body $m_{3}$ (moon) relative to the 2 nd body $m_{2}$ (planet), is varying for all variety of the moons of the planets (in Solar System) from the meaning $0.0004 \cdot 10^{-6}$ (for Proteus of Neptune) to the meaning $54.46 \cdot 10^{-6}$ (for Iapetus of Saturn), but it still remains to be negligible enough for adopting the stable moving of the effective mass $\left(\xi \cdot m_{2}+m_{3}\right)$ on quasielliptic Kepler's orbit around their common centre of masses with the 2 nd body $m_{2}$.

Equation (8) and the corresponding parameter $\xi$ play a key role in this paper. As for the physical meaning of (8), it describes the relative motion of the centre of mass of 2 nd giant-body $m_{2}$ (planet) and the centre of mass of 3rd body (moon) with the effective mass $\left(\xi \cdot m_{2}+m_{3}\right)$, which are rotating around their common centre of masses on the stable Kepler's elliptic trajectories. In case the dimensionless parameters $\xi$, $\eta \rightarrow 0$ then (8) should describe a quasi-circle motion of 3 rd body (moon) around the 2 nd body $m_{2}$ (planet).

For example, (8) refers to the classical two-body problem if $\xi$ is a constant; nevertheless, $\xi$ is fluctuating with time during orbital motion in R3BP, and hence (8) actually describes a perturbed two-body problem and its solution is nonconstant elliptic instead of fixed elliptic. As for physical explanation on the effective mass $\left(\xi \cdot m_{2}+m_{3}\right)$, it seems that $\xi \cdot m_{2}$ could be also considered as the secular part of the third-body perturbation.

As for the connection (similarities, differences, etc.) between equation of relative motion (8) and the classical perturbed two-body problem (with the main perturbation being third-body gravity), they are roughly equivalent, but the proposed ansatz is obviously an alternative approach, which could be more effective for the investigations of mutual relative motion and stability of the moons orbits in Solar System.

If the total sum of dimensionless parameters $(\xi+\eta)$ is negligible then (8) should describe a stable quasi-circle orbit of 3 rd body (moon) around the 2 nd body $m_{2}$ (planet). Let us consider the proper examples which deviate (differ) to some extent from the negligibility case $(\xi+\eta) \rightarrow 0$ above (Table 1 ) [11]:

(1) Nereid-Neptune:

$(\xi+\eta)=(35.81+0,29) \cdot 10^{-6}, \quad$ eccentricity $\mathbf{0 . 7 5 0 7}$

(2) Triton-Neptune:

$(\xi+\eta)=(0.01+210) \cdot 10^{-6}$,

eccentricity 0.000016 .

(3) Iapetus-Saturn:

$(\xi+\eta)=(\mathbf{5 4 . 4 6}+3.4) \cdot 10^{-6}, \quad$ eccentricity 0.0286 .

(4) Titan-Saturn:

$(\xi+\eta)=(2.2+240) \cdot 10^{-6}, \quad$ eccentricity 0.0288 .

(5) Io-Jupiter:

$(\xi+\eta)=(0.168+47) \cdot 10^{-6}, \quad$ eccentricity 0.0041 .

(6) Callisto-Jupiter:

$(\xi+\eta)=(14.89+58) \cdot 10^{-6}, \quad$ eccentricity 0.0074 .

(7) Ganymede-Jupiter:

$(\xi+\eta)=(2.73+79) \cdot 10^{-6}, \quad$ eccentricity 0.0013

(8) Phobos-Mars:

$(\xi+\eta)=(0,217+0,02) \cdot 10^{-6}, \quad$ eccentricity $\mathbf{0 . 0 1 5 1}$.

(9) Moon-Earth:

$(\xi+\eta)=(5,532+12,300) \cdot 10^{-6}$,

eccentricity 0.0549 .

The obvious extreme exception is the Nereid (moon of Neptune) from this scheme; Nereid orbits Neptune in the prograde direction at an average distance of 5,513,400 km, but its high eccentricity of 0.7507 takes it as close as $1,372,000 \mathrm{~km}$ and as far as $9,655,000 \mathrm{~km}$ [11].

The unusual orbit suggests that it may be either a captured asteroid or Kuiper belt object or that it was an inner moon in the past and was perturbed during the capture of Neptune's 
TABle 1: Comparison of the averaged parameters of the moons in Solar System.

\begin{tabular}{|c|c|c|c|c|c|}
\hline $\begin{array}{l}\text { Masses of the } \\
\text { planets (Solar } \\
\text { System), kg }\end{array}$ & $\begin{array}{l}\text { Ratio } m_{1} \text { (Sun) to } \\
\text { mass } m_{2} \text { (planet) }\end{array}$ & $\begin{array}{c}\text { Distance }\left|\mathbf{R}_{1,2}\right| \\
\text { (between Sun and } \\
\text { planet), AU }\end{array}$ & $\begin{array}{l}\text { Parameter } \eta \text {, ratio } \\
m_{3} \text { (moon) to mass } \\
m_{2} \text { (planet) }\end{array}$ & $\begin{array}{l}\text { Distance }\left|\mathbf{R}_{2,3}\right| \text { (between } \\
\text { moon and planet) in } \\
10^{3} \mathrm{~km}\end{array}$ & $\begin{array}{l}\text { Parameter } \\
\xi=\left(\left(m_{1} / m_{2}\right) \cdot\left(\left|\mathbf{R}_{2,3}\right|^{3} /\left|\mathbf{R}_{1,2}\right|^{3}\right)\right)\end{array}$ \\
\hline $\begin{array}{l}\text { Mercury, } \\
3.3 \cdot 10^{23}\end{array}$ & $(332,946 / 0.055)$ & $0.387 \mathrm{AU}$ & & & \\
\hline $\begin{array}{l}\text { Venus, } \\
4.87 \cdot 10^{24}\end{array}$ & $(332,946 / 0.815)$ & $0.723 \mathrm{AU}$ & & & \\
\hline $\begin{array}{l}\text { Earth, } \\
5.97 \cdot 10^{24} \\
\end{array}$ & $\begin{array}{c}1 \text { Earth = } \\
332,946 \mathrm{~kg}\end{array}$ & $\begin{array}{c}1 \mathrm{AU}= \\
149,500,000 \mathrm{~km}\end{array}$ & $12,300 \cdot 10^{-6}$ & 383.4 & $\begin{array}{l}\text { Moon: } \\
5,532 \cdot 10^{-6}\end{array}$ \\
\hline $\begin{array}{l}\text { Mars, } \\
6.42 \cdot 10^{23}\end{array}$ & $(332,946 / 0.107)$ & $1.524 \mathrm{AU}$ & $\begin{array}{l}\text { (1) Phobos: } \\
0.02 \cdot 10^{-6} \\
\text { (2) Deimos: } \\
0.003 \cdot 10^{-6}\end{array}$ & $\begin{array}{l}\text { (1) Phobos: } \\
\text { 9.38 } \\
\text { (2) Deimos: } \\
\text { 23.46 }\end{array}$ & $\begin{array}{l}\text { (1) Phobos: } \\
0.22 \cdot 10^{-6} \\
\text { (2) Deimos: } \\
3.4 \cdot 10^{-6}\end{array}$ \\
\hline $\begin{array}{l}\text { Jupiter, } \\
1.9 \cdot 10^{27}\end{array}$ & $(332,946 / 317.8)$ & $5.2 \mathrm{AU}$ & $\begin{array}{l}\text { (1) Ganymede: } \\
\mathbf{7 9} \cdot \mathbf{1 0}^{-\mathbf{6}} \\
\text { (2) Callisto: } \\
\mathbf{5 8} \cdot \mathbf{1 0}^{-\mathbf{6}} \\
\text { (3) Io: } \\
\mathbf{4 7} \cdot \mathbf{1 0}^{-6} \\
\text { (4) Europa: } \\
25 \cdot 10^{-6}\end{array}$ & $\begin{array}{l}\text { (1) Ganymede: } \\
\mathbf{1 , 0 7 0} \\
\text { (2) Callisto: } \\
\mathbf{1 , 8 8 3} \\
\text { (3) Io: } \\
422 \\
\text { (4) Europa: } \\
671\end{array}$ & $\begin{array}{l}\text { (1) Ganymede: } \\
2.73 \cdot 10^{-6} \\
\text { (2) Callisto: } \\
\text { 14.89 } 10^{-6} \\
\text { (3) Io: } \\
0.17 \cdot 10^{-6} \\
\text { (4) Europa: } \\
0.67 \cdot 10^{-6}\end{array}$ \\
\hline $\begin{array}{l}\text { Saturn, } \\
5.69 \cdot 10^{26}\end{array}$ & $(332,946 / 95.16)$ & $9.54 \mathrm{AU}$ & $\begin{array}{l}\text { (1) Titan: } \\
\text { 240 } \cdot \mathbf{1 0}^{-\mathbf{6}} \\
\text { (2) Rhea: } \\
\text { 4.1 } \cdot 10^{-6} \\
\text { (3) Iapetus: } \\
\text { 3.4 } \cdot 10^{-6} \\
\text { (4) Dione: } \\
\text { 1.9 } \cdot 10^{-6} \\
\text { (5) Tethys: } \\
\text { 1.09 } \cdot 10^{-6} \\
\text { (6) Enceladus: } \\
0.19 \cdot 10^{-6} \\
\text { (7) Mimas: } \\
0.07 \cdot 10^{-6}\end{array}$ & $\begin{array}{l}\text { (1) Titan: } \\
\text { 1,222 } \\
\text { (2) Rhea: } \\
\text { 527 } \\
\text { (3) Iapetus: } \\
\text { 3,561 } \\
\text { (4) Dione: } \\
\text { 377 } \\
\text { (5) Tethys: } \\
\text { 294.6 } \\
\text { (6) Enceladus: } \\
\text { 238 } \\
\text { (7) Mimas: } \\
\text { 185.4 }\end{array}$ & $\begin{array}{l}\text { (1) Titan: } \\
2.2 \cdot 10^{-6} \\
\text { (2) Rhea: } \\
0.18 \cdot 10^{-6} \\
\text { (3) Iapetus: } \\
\mathbf{5 4 . 4 6} \cdot \mathbf{1 0}^{-6} \\
\text { (4) Dione: } \\
0.07 \cdot 10^{-6} \\
\text { (5) Tethys: } \\
0.03 \cdot 10^{-6} \\
\text { (6) Enceladus: } \\
0.016 \cdot 10^{-6} \\
\text { (7) Mimas: } \\
0.008 \cdot 10^{-6}\end{array}$ \\
\hline $\begin{array}{l}\text { Uranus, } \\
8.69 \cdot 10^{25}\end{array}$ & $(332,946 / 14.37)$ & 19.19 AU & $\begin{array}{l}\text { (1) Titania: } \\
40 \cdot 10^{-6} \\
(2) \text { Oberon: } \\
35 \cdot 10^{-6} \\
\text { (3) Ariel: } \\
16 \cdot 10^{-6} \\
(4) \text { Umbriel: } \\
\text { 13.49 } \cdot 10^{-6} \\
(5) \text { Miranda: } \\
0.75 \cdot 10^{-6} \\
\end{array}$ & $\begin{array}{l}\text { (1) Titania: } \\
436 \\
\text { (2) Oberon: } \\
584 \\
\text { (3) Ariel: } \\
191 \\
\text { (4) Umbriel: } \\
\text { 266.3 } \\
\text { (5) Miranda: } \\
\text { 129.4 }\end{array}$ & $\begin{array}{l}\text { (1) Titania: } \\
0.08 \cdot 10^{-6} \\
\text { (2) Oberon: } \\
0.2 \cdot 10^{-6} \\
\text { (3) Ariel: } \\
0.01 \cdot 10^{-6} \\
\text { (4) Umbriel: } \\
0.019 \cdot 10^{-6} \\
(5) \text { Miranda: } \\
0.002 \cdot 10^{-6} \\
\end{array}$ \\
\hline $\begin{array}{l}\text { Neptune, } \\
1.02 \cdot 10^{26}\end{array}$ & $(332,946 / 17.15)$ & $30.07 \mathrm{AU}$ & $\begin{array}{l}\text { (1) Triton: } \\
\mathbf{2 1 0} \cdot \mathbf{1 0}^{-\mathbf{6}} \\
\text { (2) Proteus: } \\
0.48 \cdot 10^{-6} \\
\text { (3) Nereid: } \\
0.29 \cdot 10^{-6}\end{array}$ & $\begin{array}{l}\text { (1) Triton: } \\
355 \\
\text { (2) Proteus: } \\
118 \\
\text { (3) Nereid: } \\
\mathbf{5 , 5 1 3}\end{array}$ & $\begin{array}{l}\text { (1) Triton: } \\
0.01 \cdot 10^{-6} \\
\text { (2) Proteus: } \\
0.0004 \cdot 10^{-6} \\
\text { (3) Nereid: } \\
35.81 \cdot 10^{-6}\end{array}$ \\
\hline
\end{tabular}

largest moon Triton [11]. One could suppose that the orbit of Nereid should be derived preferably from the assumptions of R4BP (the case of restricted four-body problem) or more complicated cases.

As we can see from consideration above, in case of Earth's Moon, such dimensionless key parameters increase simultaneously to the crucial meanings $\xi=0.0055$ and $\eta=0.0123$, respectively, $(\xi+\eta)=0.0178$. It means that the orbit for relative motion of the moon in regard to the Earth could not be considered as quasi-elliptic orbit and should be considered as nonconstant elliptic orbit with the effective mass $\left(\xi \cdot m_{2}+\right.$ $m_{3}$ ) placed in the centre of mass for the moon.

As we know $[3,4]$, the elements of that elliptic orbit depend on the position of the common centre of masses for 
3rd small body (moon) and the planet (Earth). But such a position of their common centre of mass should obviously differ for the real mass $m_{3}$ and the effective mass $\left(\xi \cdot m_{2}+m_{3}\right)$ placed in the centre of mass of the 3 rd body (moon). So, the elliptic orbit of motion of the moon derived from the assumptions of R3BP should differ from the elliptic orbit which could be obtained from the assumptions of R2BP (the case of restricted two-body problem: it means mutual moving of 2 gravitating masses without the influence of other central forces).

As for the meanings of the terms quasi-elliptic, quasicircle, and nonconstant elliptic, "quasi" means that the main orbital elements of the orbit of moon around the planet still remain approximately the same without essential alterations (due to negligible influence of moon's gravity in a frame of the R3BP), but the term "nonconstant elliptic" means that (8) describes actually a perturbed two-body problem and its solution is nonconstant elliptic instead of fixed elliptic.

\section{Remarks about the Eccentricities of the Orbits}

According to the definition [11], the orbital eccentricity of an astronomical object is a parameter that determines the amount by which its orbit around another body deviates from a perfect orbit:

$$
e=\sqrt{1+\frac{2 \varepsilon h^{2}}{\mu^{2}}},
$$

where $\varepsilon$ is the specific orbital energy, $h$ is the specific angular momentum, $\mu$ is the sum of the standard gravitational parameters of the bodies, and $\mu=\gamma \cdot m_{2} \cdot(1+\xi+\eta)$; see (8).

The specific orbital energy equals the constant sum of kinetic and potential energy in a 2-body ballistic trajectory [11]:

$$
\varepsilon=-\frac{\mu}{2 a}=\text { const; }
$$

here $a$ is the semimajor axis. For an elliptic orbit, the specific orbital energy is the negative of the additional energy required to accelerate a mass of one kilogram to escape velocity (parabolic orbit).

Thus, assuming $\xi=\xi(t)$, we should obtain the following from the equality above:

$$
\begin{aligned}
& -\frac{\gamma \cdot m_{2}(1+\xi(t)+\eta)}{2 a(t)}=\mathrm{const}, \\
& \Longrightarrow a(t)=a_{0} \cdot(1+\xi(t)+\eta),
\end{aligned}
$$

where $\xi(t)$ is the periodic function depending on timeparameter $t$, which is slowly varying during all the timeperiods from the minimal meaning $\xi_{\min }>0$ to the maximal meaning $\xi_{\text {max }}$, preferably $\left(\xi_{\max }-\xi_{\min }\right) \rightarrow 0$.

Besides, we should note that, in an elliptical orbit, the specific angular momentum $h$ is twice the area per unit time swept out by a chord of ellipse (i.e., the area which is totally covered by a chord of ellipse during its motion per unit time, multiplied by 2) from the primary to the secondary body [11], according to Kepler's 2nd law of planetary motion.

Since the area of the entire orbital ellipse is totally swept out in one orbital period, the specific angular momentum $h$ is equal to twice the area of the ellipse divided by the orbital period, as represented by

$$
h=b \sqrt{\frac{\gamma(1+\xi+\eta) \cdot m_{2}}{a}},
$$

where $b$ is the semiminor axis. So, from (20) we should obtain that, for the constant specific angular momentum $h$, the semiminor axis $b$ should be constant also.

Thus, we could express the components of elliptic orbit as follows:

$$
\begin{aligned}
& x(t)=a_{0} \cdot(1+\xi(t)+\eta) \cdot \cos t, \\
& y(t)=b \cdot \sin t
\end{aligned}
$$

which could be schematically imagined as it is shown in Figures 1(a), 1(b), and 1(c).

As for the chosen parameters in Figures 1(a), 1(b), and 1(c), meanings of the parameter $a_{0} \cdot \eta$ are varying in the range from 0.0123 (Figure 1(a)) to 10.123 (Figure 1(b)) and 49.5 (Figure $1(\mathrm{c})$ ); parameter $a_{0} \cdot \xi(t)$ is varying in the range from $0.0055 \cdot(0.9+0.1 \cdot \sin t)$ (Figure $1(\mathrm{a}), b=1)$ to $0.55 \cdot(0.9+$ $0.1 \cdot \sin t)$ (Figure $1(\mathrm{~b}), b=10)$ and $100 \cdot(1.0+0.5 \cdot \sin t)$ (Figure 1(c), $b=150)$. Obviously, we can see that the orbit of moon at the end of Figures 1(a), 1(b), and 1(c) quite differs from the elliptic one.

\section{Conclusion}

We have considered the equations of motion of three-body problem in a Lagrange form (for the relative motions of 3 bodies in regard to each other). Analyzing such a system of equations, we explore the case of moon's motion of negligible mass $m_{3}$ around the 2 nd of two giant-bodies $m_{1}, m_{2}$, the mass of which is assumed to be less than the mass of central body $m_{2}$. Besides, only the natural satellites which are massive enough to have achieved hydrostatic equilibrium have been considered. Twenty-two of such midsized natural satellites for planets of Solar System, including Earth's Moon, are known; see Table 1.

The elegant derivation of a key parameter $\xi$ that determines the character of the moving of the moon relative to the planet has been proposed.

We also obtain that the equations of motion R3BP should describe the relative mutual motion of the centre of mass of 2nd giant-body $m_{2}$ (planet) and the centre of mass of 3rd body (moon) with additional effective mass $\xi \cdot m_{2}$ placed in that centre of mass $\left(\xi \cdot m_{2}+m_{3}\right)$, where $\xi$ is the dimensionless dynamical parameter (nonconstant, but negligible). Thus, they should be rotating around their common centre of masses on Kepler's elliptic orbits. So, the case R3BP of " 3 -body problem" for the moon's orbit was elegantly reduced to the case R2BP of "2-body problem" (the last one is known to be stable for the relative motion of "planet-satellite" pairs $[3,4])$. 


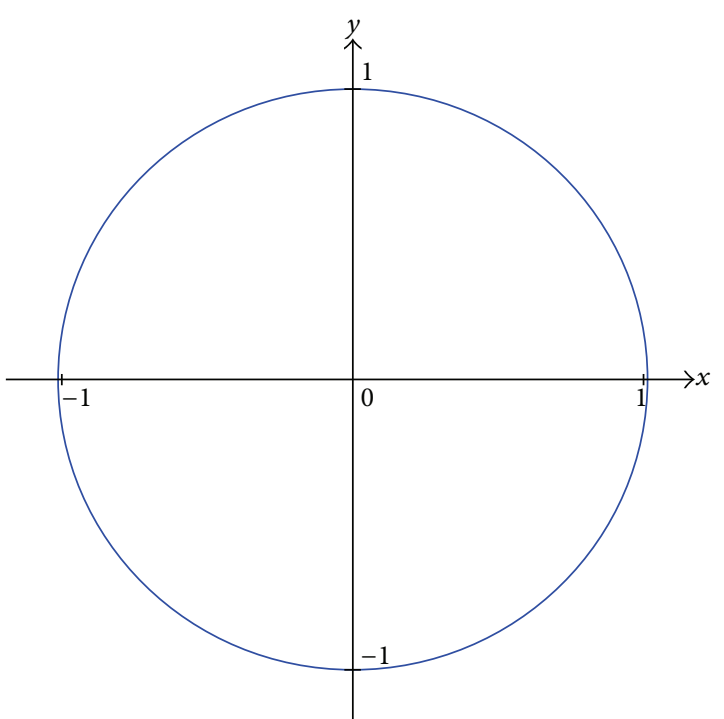

(a)

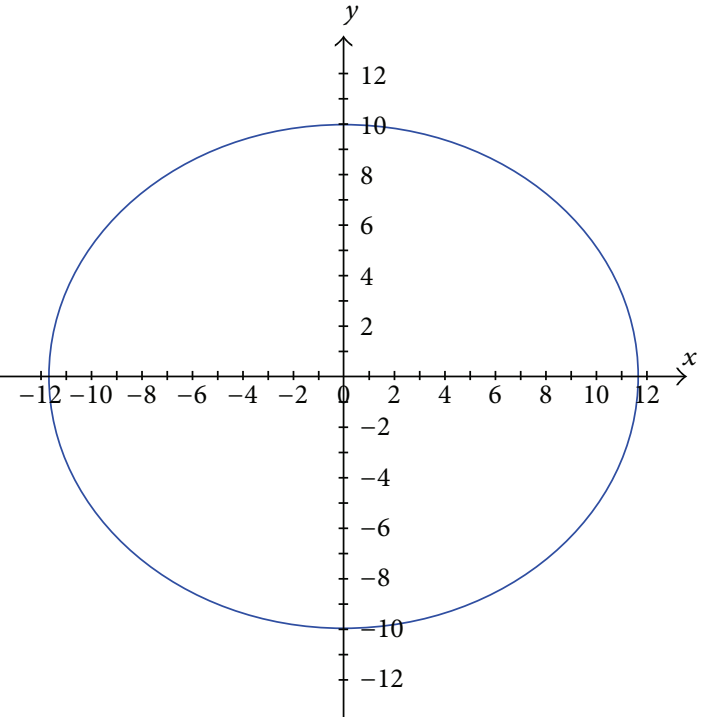

(b)

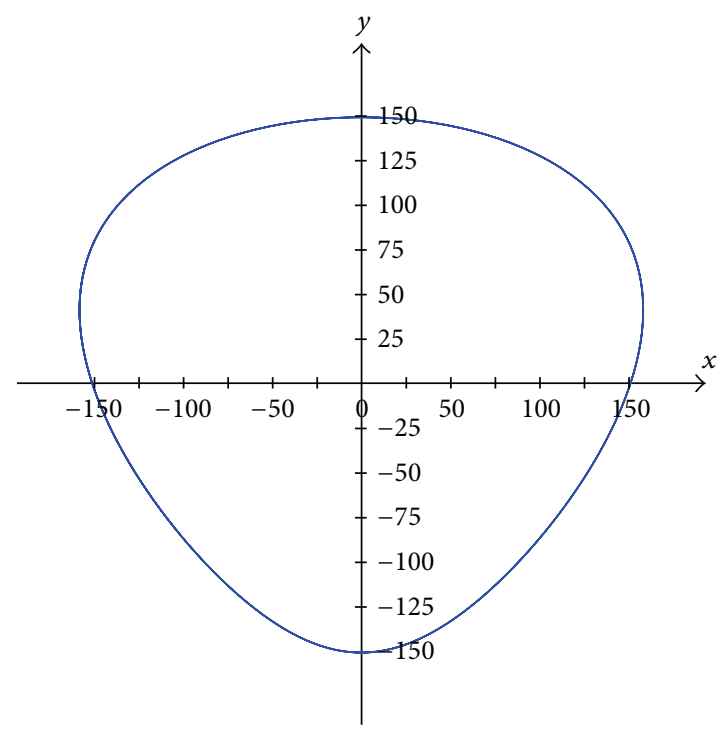

(c)

Figure 1: Orbits of the moon, schematically imagined.

For negligible effective mass $\left(\xi \cdot m_{2}+m_{3}\right)$ it gives equations of motion which should describe a quasi-elliptic orbit of 3rd body (moon) around the 2nd body $m_{2}$ (planet) for most of the moons of the planets in Solar System. But the orbit of Earth's Moon should be considered as nonconstant elliptic motion for the effective mass $0.0178 \cdot m_{2}$ placed in the centre of mass for the 3rd body (moon). The position of their common centre of masses should obviously differ for the real mass $m_{3}=0.0123 \cdot m_{2}$ and for the effective mass $(0.0055+$ $0.0123) \cdot m_{2}$ placed in the centre of mass of the moon.

\section{Conflict of Interests}

The author declares that there is no conflict of interests regarding the publication of this paper.

\section{Acknowledgment}

The author devotes this paper to his wife for her heart love which is the only source of his scientific spirit of creation.

\section{References}

[1] V. I. Arnold, Mathematical Methods of Classical Mechanics, Springer, New York, NY, USA, 1978.

[2] J. Lagrange, OEuvres, vol. 6, Gautier-Villars, Paris, France, 1873, edited by M. J. A. Serret.

[3] V. Szebehely, Theory of Orbits. The Restricted Problem of Three Bodies, Yale University, New Haven, Conn, USA; Academic Press, London, UK, 1967. 
[4] G. N. Duboshin, "Nebesnaja mehanika. Osnovnye zadachi i metody," in Handbook for Celestial Mechanics, Nauka, Moscow, Russia, 1968, (Russian).

[5] H. Bruns, "Über die integrale des Vielkörper-problems," Acta Mathematica, vol. 11, no. 1-4, pp. 25-96, 1887.

[6] Shankaran, J. P. Sharma, and B. Ishwar, "Equilibrium points in the generalised photogravitational non-planar restricted three body problem," International Journal of Engineering, Science and Technology, vol. 3, no. 2, pp. 63-67, 2011.

[7] Y. A. Chernikov, “The photogravitational restricted three-body problem," Soviet Astronomy, vol. 14, p. 176, 1970.

[8] J. Singh and O. Leke, "Stability of the photogravitational restricted three-body problem with variable masses," Astrophysics and Space Science, vol. 326, no. 2, pp. 305-314, 2010.

[9] S. V. Ershkov, "The Yarkovsky effect in generalized photogravitational 3-body problem," Planetary and Space Science, vol. 73, no. 1, pp. 221-223, 2012.

[10] E. Kamke, Hand-Book for ODE, Science, Moscow, Russia, 1971.

[11] S. S. Sheppard, The Giant Planet Satellite and Moon Page, Departament of Terrestrial Magnetism at Carniege Institution for Science, 2015, http://en.wikipedia.org/wiki/Solar_System. 

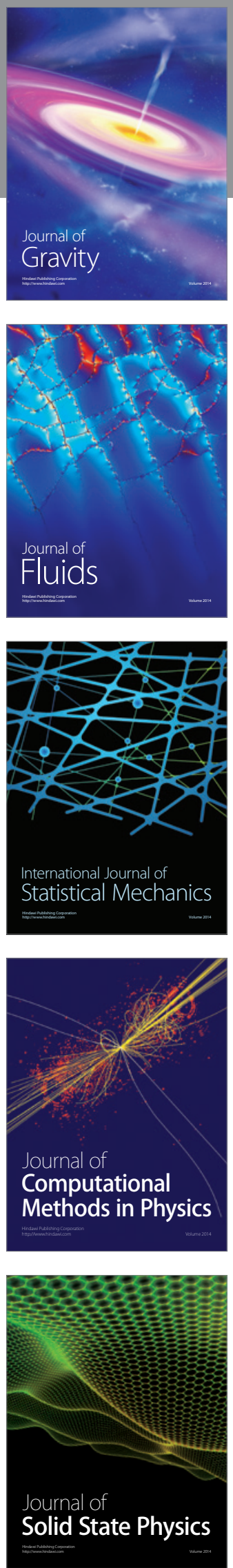

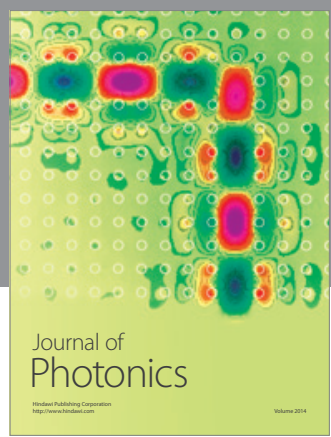

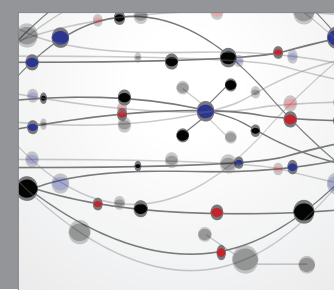

The Scientific World Journal

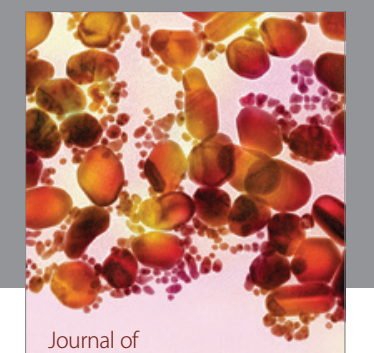

Soft Matter
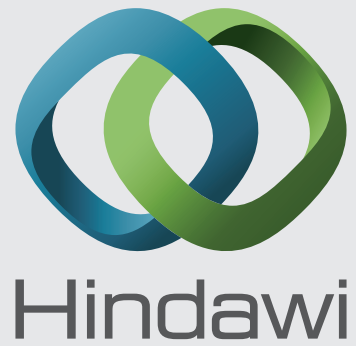

Submit your manuscripts at

http://www.hindawi.com
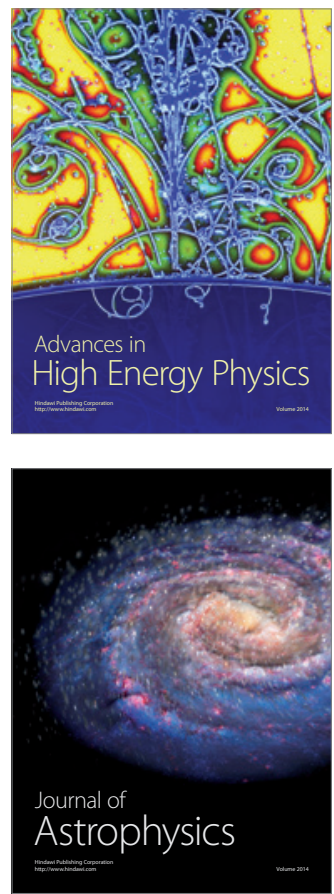
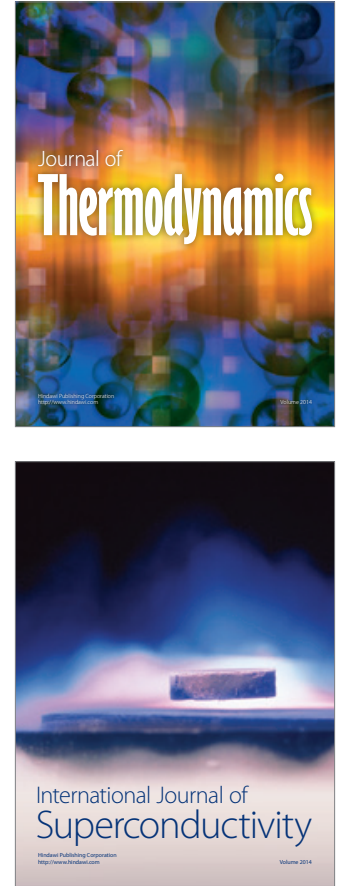
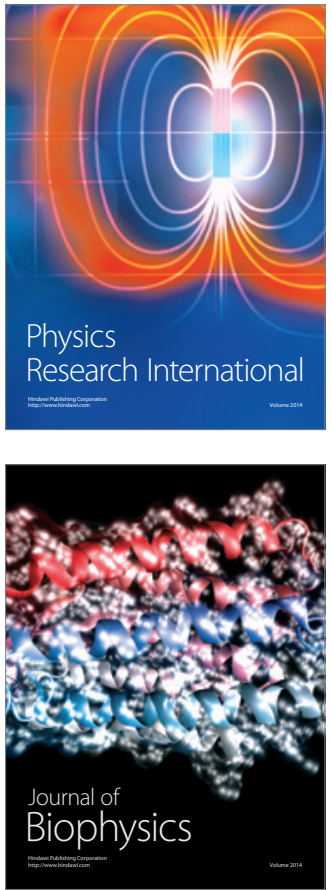
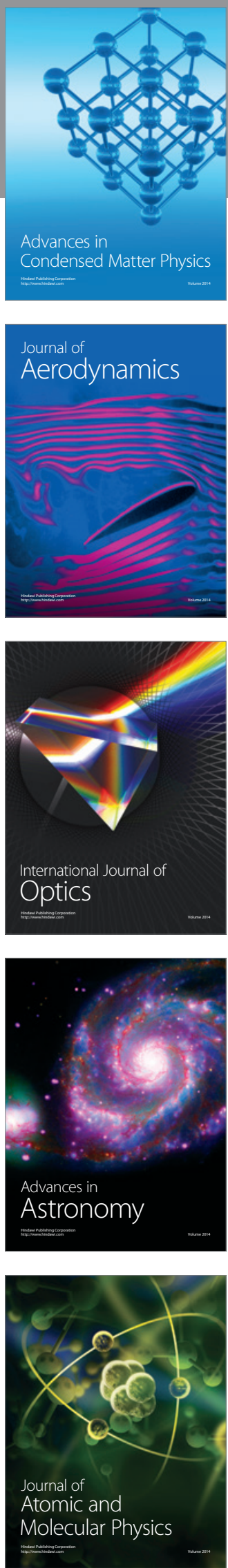